\title{
Fremdsprachendidaktik anhand von Literatur: Reflexion über Stereotype
}

\author{
Gesa Singer (Flensburg)
}

\begin{abstract}
Stereotypes are often based on concepts of mentalities and 'images of the self' and the 'other'. This is the way they appear in language. In modern didactics of foreign language the focus is mostly set on cultural contrast. Meanwhile a more profound analysis and reflexion on stereotypes is lacking.

This piece of work intends to illustrate, based on practical examples (in German as a foreign language), how the use of literature can contribute to a critical and productive work and discussion on stereotypes.

Recent research on intercultural didactics of foreign languages as well as empirical studies are applied in this part of a model concept of teaching literature through dialogue and interaction. Students learn to comment thoughtfully on 'self' and 'foreign' imagery. It is, from here on, a didactical proposal for different intercultural settings.
\end{abstract}

\section{$1 \quad$ Einleitung}

Stereotype äußern sich oft sprachlich und die ihnen zugrunde liegenden Vorstellungen sind an Mentalitäten sowie die Konstruktion von Fremd- und Eigenbildern gebunden. Die moderne Fremdsprachendidaktik greift zwar vielfach kulturkontrastive Modelle auf, bemüht sich aber selten um eine differenzierte Auseinandersetzung und Reflexion über Stereotype. Insbesondere deren Rolle im Rahmen der interkulturellen Kommunikation bleibt oft unklar. Interkulturelle Kommunikation ist in einer globalisierten Welt Voraussetzung für gelingende Kooperation.

Die bei interkultureller Kommunikation geforderte Selbstreflexion bezieht sich im übrigen nicht nur auf individuelle Motive, Vorurteile etc., sondern auf die eigenen Kulturmuster einschließlich der in der eigenen Gesellschaft oder Gruppe gehandelten Stereotypen und Vorurteile.

(Auernheimer 2005: 12)

Mein Beitrag soll anhand von Beispielen zeigen, wie der Einsatz von Literatur im Fremdsprachenunterricht (speziell DaF) zur kritischen und produktiven Beschäftigung mit Stereotypen anregen kann, indem eine Reflexion über Vorstellungen von „Fremd“ und „Eigen“ in einem interkulturellen Kommunikationsprozess stattfindet.

Ausgehend von neueren Untersuchungen zur interkulturellen Fremdsprachendidaktik wird mit Blick auf Stereotype ein Ausschnitt meines Habilitationsprojekts zur interkulturellen Litera-

Linguistik online 79, 5/2016 - http://dx.doi.org/10.13092/lo.79.3341

CC by 3.0 
turvermittlung dargestellt. Es soll (anhand meiner Lehrerfahrung als DAAD Lektorin 20072011 an der Aristoteles Universität Thessaloniki) modellhaft gezeigt werden, wie in einem dialogischen Prozess bei der handlungsorientierten Auseinandersetzung mit ausgewählten Literaturausschnitten Konstrukte von ,Fremd' und ,Eigen' kritisch hinterfragt und Prozesse von Stereotypenbildung einerseits sowie Möglichkeiten der Selbstreflexion andererseits bewusst gemacht werden können.

Im Hinblick auf weitere Anwendungsbereiche wird schließlich die Übertragbarkeit eines solchen Unterrichtsmodells auf andere kulturelle Kontexte erörtert.

\section{$2 \quad$ Stereotype}

Seit Walter Lippmann 1922 den Ausdruck Stereotyp als Metapher aus der Druckersprache entlehnt hat, hat sich die Sozialpsychologie und Linguistik intensiv damit befasst, und daneben ist er auch als Alltagsvokabel in aller Munde. Insbesondere in der Fremdsprachendidaktik interessiert man sich für Stereotype, weil neben der reinen Sprachvermittlung oft auch die Auseinandersetzung mit kulturellen Eigenarten des Zielsprachenlandes und ihrer Vertreter eine Aufgabe des Unterrichts bildet. Die Imagologie wiederum widmet sich in der Literaturwissenschaft der Erforschung von Bildern von anderen Ländern (cf. Pümpel-Mader 2010: 9).

Stereotype beziehen sich häufig verallgemeinernd auf Nationen oder Kulturen sowie Angehörige bestimmter Gruppen, seltener auf einzelne Individuen (z. B. „Die Deutschen sind fleiBig“; „Italiener gestikulieren viel“" „Latinos haben Rhythmus im Blut“ etc.). Mit solchen Zuschreibungen versucht man die Zugehörigkeit zur eigenen kulturellen Gruppe (mittels Autostereotypen=Selbstzuschreibungen: „Wir sind so“) hervorzuheben und sich von anderen (mittels Heterostereotypen=Fremdzuschreibungen: „Die sind anders“") vergleichend abzugrenzen. Doch ist häufig zu beobachten, wie Menschen im alltäglichen Diskurs bemüht sind, Ausnahmen von solchen Stereotypen zu formulieren (z. B. „Ich mag die Türken nicht, weil [...] aber meine Freundin ist Türkin, denn sie ist keine typische Türkin“" o. ä.).

Unter anderem sind in den fünfziger Jahren des vergangenen Jahrhunderts auch Arbeiten zu wechselseitigen Völker-Bildern auf der Grundlage operationalisierender Verfahren entstanden, die ,Auto'- und ,Hetero'-Stereotype unterscheiden und miteinander vergleichen.

(Florack 2007: 33)

Demnach beruhen Stereotype auf Zuschreibungen von ,fremd' und ,eigen' und den damit jeweils assoziierten Kriterien oder Eigenschaften. Zur Begriffsbestimmung nur so viel:

Unter dem Einfluss der ,Social-Cognition“ -Forschung beziehen heutzutage die meisten Experten den „Begriff des Stereotyps“ auf „kognitive Prozesse der Abwertung“, also die Ebene negativer Einstellungen bzw. Antipathien. Dabei darf deren Bezug zu diskriminierendem Handeln keineswegs eindeutig als Ursache-Wirkung-Relation verstanden werden, sondern muß [sic!], abhängig von Personen und gesellschaftlichem Kontext, multifaktoriell erklärt werden.

(Florack 2007: 35)

Wie es zu einer Äußerung über die Deutschen wie der folgenden kommen kann, müsste also multifaktoriell untersucht werden: Es handele sich um „,einförmige, supernationalistische Blonde, die lärmend über die Strände herfallen."“ (Pümpel-Mader 2010: 17, die Worte des 
italienischen Wirtschaftssekretärs von 2003 zitierend.) Man könnte also fragen, in welchem gesellschaftlichen und historischen Kontext die Deutschen als Touristen im jeweiligen Gastgeberland mit Vokabeln assoziiert werden, die eher an Krieg und Territorialkämpfe (,supernationalistisch“; „herfallen“) erinnern und einen unangenehmen Eindruck verursachen (,einförmig“; ,lärmend“) und welche weiteren situativen Faktoren solche Wahrnehmung und Zuschreibung begünstigen.

In einer Synopse von Schugk (2004: 72-73) treten einige der Hetero- und Autostereotype innerhalb Europas zutage, welche durchaus auch als ein humoristischer bzw. kritischer Reflex auf historische Prägungen und Kommunikationsmechanismen angesehen werden können. Dazu sei einmal betrachtet, was ,der Deutsche‘ über ,den Dänen` denkt:

„Der Italiener des Nordens, leider erwidern sie die deutsche Liebe ebenso wenig wie die Italiener."

Umgekehrt ,der Däne“ über den ,Deutschen`:

„Tun gern etwas Verbotenes (Strandburgen bauen, zu schnell fahren, Weltkriege anfangen)“.

Dagegen ,Dänen“ über sich selbst:

„Lustig, humorvoll, mit aufrichtiger Liebe zu bunten Speisen, Aquavit und Steuerhinterziehung";

Die ,Deutschen` über sich selbst:

„Die ungeliebte Nation. Nach schlimmer Straftat nur auf Bewährung entlassen.“

(die drei zuletzt genannten Zitate von Schugk 2004: 72-73)

Neben einer Tendenz zur Verharmlosung lässt sich an diesen fantasievollen Beispielen ablesen, dass die Deutschen auto- und heterostereotyp mit den zwei Weltkriegen in Verbindung gebracht werden und die Dänen als hedonistisch und vergleichsweise harmlos gelten. Ohne einen Anspruch auf Allgemeingültigkeit zu erheben, ist darin wohl auch ein Reflex der wechselseitigen Wahrnehmung dieser beiden Kulturen in den Medien und Lehrwerken der vergangenen Dekaden zu beobachten (cf. Hallsteinsdóttir 2012; Lammers 2014).

Stereotype dienen der diskursiven Herstellung von (nationalen) Identitäten, können unter Umständen eine orientierende Funktion haben und müssen nicht ausschließlich negativ, sondern können auch positiv konnotiert sein. Stereotype können Bestandteil des kulturellen Wissens sein, weisen eine relativ geringe Tendenz zur Veränderlichkeit auf, können sich starr verfestigen und sind aber grundsätzlich unabhängig von individuellen Einstellungen, Meinungen und Vorurteilen. Sie äußern sich in internalisierten Bildern oder verkürzten sprachlichen Formen und Gemeinplätzen; oft basieren sie auf groben Verallgemeinerungen und dienen in der Kommunikation als Versatzstücke, die m. E. häufig eine erste und oberflächliche, teils auch ironische Verständigung über Phänomene herstellen sollen, für deren differenzierte Betrachtung das Interesse, die Kompetenz der Teilnehmer oder die Zeit punktuell nicht ausreichen (cf. Hallsteinsdóttir 2012; Lammers 2014). Solche Verkürzungen zu entschlüsseln kann allerdings problematisch werden, wenn hinter der Kurzform die Differenziertheit der historischen und kulturellen Realitäten überhaupt nicht mehr erkannt oder absichtlich verzerrt wird (cf. Addicks et al. 2012). 


\section{$3 \quad$ Fremd und Eigen}

Innerhalb der Germanistik hat der Kulturbegriff besonders durch die interkulturelle Germanistik eine Aufwertung erfahren, da angeregt durch die Perspektive der Auslandsgermanistik sowie mehrerer Bezugswissenschaften die Mehrdimensionalität von Kultur in den Blick genommen und intensiv diskutiert wurde. Leider ist diese Diskussion jedoch auch zuweilen auf stereotype Zuschreibungen und Kulturkontraste zurückgefallen.

Die von Reimer (2005) zusammengefasste Kritik an der Studie von Geert Hofstede über kulturelle Dimensionen hat nicht zuletzt gezeigt, dass es notwendig ist, Kultur als etwas Bewegliches, Veränderbares zu verstehen, das nicht starr und fixiert ist. Darüber hinaus müssten individuelle Variablen (Geschlecht, Status, biographische Faktoren) in eine ernstzunehmende Studie über Verhaltensdimensionen und ihre kulturelle Gebundenheit mit einbezogen werden. Auf dieser Basis könnte man sich neu über kulturelle Differenzen unterhalten, ohne in überkommene Zuschreibungen zu verfallen.

Hier geht es u.a. um die Beschreibung und Sichtbarmachung von kulturell bedingten sozialpsychologischen Zuschreibungsprozessen, die das komplexe Beziehungsgeflecht von ,fremd ‘ und , eigen', und was damit jeweils assoziiert wird, analysieren. Diese Prozesse dienen zur Wahrnehmung und Schaffung von Gruppenidentitäten durch Ab- und Ausgrenzungen und haben oft polemischen Charakter, wie z. B. der Ausdruck Überfremdung.

Indem interkulturelle Germanistik den Begriff Fremdheit zu einem ihrer Rahmenbegriffe macht, übernimmt sie die [...] Spezifika, die das Wort fremd im deutschen Sprachgebrauch aufweist: die Mehrdeutigkeit eines nicht klar unterschiedenen Bedeutungskontinuums, seine Unbestimmtheit durch deiktische und relationale Verweisstruktur und seinen Negationscharakter.

(Albrecht 2003: 234)

Andererseits kann die Sehnsucht oder Suche nach dem ,Fremden' auch positiv besetzt sein und positive Klischees und Stereotype beinhalten: „Kennst du das Land, wo die Zitronen blüh'n..." Was Johann Wolfgang von Goethe Mignon in Wilhelm Meisters Lehrjahre (Hamburger Ausgabe, Bd. 7, S. 145) singen lässt, ist in bildungsbürgerlichen Kreisen als Sehnsuchtslied nach südlichen Gefilden sprichwörtlich geworden. Es wird deutlich, dass es sich bei positiven wie negativen Stereotypen um affektive Deutungsmuster handelt, die zwar in den intellektuellen Diskurs einfließen mögen, letztlich aber vorwiegend emotionspsychologischen Ursprung haben. Eine der Überlegungen bei der Beschäftigung mit ,fremd ‘ und ,eigen ‘ ist, die Frage, ob man aus seinem eigenkulturellen Traditionszusammenhang heraustreten und sich vom Mechanismus der Selbst- und Fremdzuschreibungen überhaupt frei machen könne: Entsprechend schließt Scheiffele die Fragen an:

Ist das überhaupt möglich? Drücken nicht Wendungen wie „keiner kann aus seiner Haut“, „niemand kann über seinen Schatten springen“ die allgemeine Erfahrung aus, daß es abgesehen von mystischer Schau, keinerlei unvermittelt-unmittelbares Verstehen gibt, daß man also sein Vorverständnis nirgends und nie los wird?

(Scheiffelle 2000: 34) 


\section{$4 \quad$ Literaturdidaktik im Fremdsprachenunterricht}

In meiner Habilitationsschrift beleuchte ich einen Aspekt der Literaturdidaktik, der kommunikativ und rezeptionsästhetisch ausgerichtet ist, und sich, angelehnt an die rezeptionsästhetische Literaturdidaktik, nicht an vorgegebenen Deutungsmustern orientiert:

Die rezeptionsästhetische Literaturdidaktik überwindet den formalistischen Literaturbegriff, indem sie aufzeigt, dass die Rezeption literarischer Texte etwas mit der Lebenserfahrung der Lernenden zu tun hat und dass es auf deren Tätigkeiten beim Lesen literarischer Texte ankommt.

(Bredella 2004: 33)

Voraussetzung ist dabei die Überlegung, dass die teilnehmerorientierte diskursive Verständigung über Literatur und ihre möglichen Interpretationen und persönlichen Assoziationen besonders in interkulturellen Zusammenhängen zu einem erweiterten und mehrperspektivischen Verständnis von kulturellen Prägungen und Differenzen beitragen kann.

Absolutes Verstehen ist hierbei möglicherweise nicht zu erreichen, aber eine Verständigung über unterschiedliche Perspektiven, eine mögliche Perspektivenübernahme und Erweiterung des Deutungshorizontes. Auch Bredella (2008: 67-86) - einer der im theoretischphilosophischen Sinne sehr weit gehenden Forscher - fragt mit einer gewissen Skepsis „Ist interkulturelles Verstehen mit literarischen Texten möglich?“

Die Rolle von Kulturalität erfährt dabei in der Literaturdidaktik eine besondere Aufwertung:

In den 1980er Jahren jedoch erfuhr schließlich die lange Zeit vernachlässigte Liaison zwischen ,Sprache' und ,Kultur' sowie die kulturelle Gebundenheit sprachlicher Handlungen, ein wichtiges Merkmal des heutigen interkulturellen Fremdsprachenunterrichts, einen entscheidenden Schub.

(Eberhardt 2013: 16)

Daher ist auch in der Literaturdidaktik des Fremdsprachenunterrichts die Bewusstmachung dieser sprachlich vermittelten kulturellen Gebundenheit ein bedeutsamer Bestandteil.

Eine der unverkennbaren Realitäten in unserer modernen Lebenswelt sind allerdings Migration und die Pluralität von Kulturen, die nicht zuletzt auch Auswirkungen auf den gesellschaftlichen, politischen und kulturellen Diskurs haben. Dies ist einer der Gründe, weshalb die Vermittlung zwischen mindestens zwei Kulturen als ,Interkulturalität' Einzug in die Wirtschaft, die Politik, die Kommunikationswissenschaft, und schließlich in didaktische Überlegungen genommen hat.

Die auch heute noch gängige Verwendung des ,Interkulturellen' in fremdsprachendidaktischen Diskursen, wie auch seine Aufnahme in Lehrpläne und Lernmaterialien des Fremdsprachenunterrichts, wurde in erster Linie begünstigt durch gesellschaftliche Faktoren wie die wachsende Migrationsbewegungen innerhalb Europas, die stete Vergrößerung des Anteils außereuropäischer Migranten sowie die demografisch bedingte Erhöhung des Migrantenanteils innerhalb der Bundesrepublik Deutschland.

(Eberhardt 2013: 17-18)

Insbesondere im Zusammenhang mit der Didaktik von Fremdsprachenunterricht ist die Vermittlung von Literatur, und zunehmend von interkultureller Literatur (die zunächst insbesondere den Phänomenen ,Fremdsein', ,Migration“ und ,Kulturunterschiede“ Raum gab), in den vergangenen Dekaden verstärkt in den Blick genommen worden: 
Konkretisiert wird diese Perspektive interkulturellen fremdsprachlichen Lernens bislang bevorzugt im Kontext von Ansätzen, die sich mit der Funktion von Texten im Unterricht befassen.

(Gogolin 2007: 100)

Eines der markantesten Bildungsziele der aktuellen Fremdsprachendidaktik ist das interkulturelle Lernen. Interkulturelle Literaturvermittlung sollte demnach unterschiedliche kulturelle Deutungsmuster im Blick haben, Stereotype aufdecken und die Reflexion darüber befördern helfen:

Interkulturelle Kompetenz ist seit einigen Jahren einer der wichtigsten Begriffe in den fremdsprachendidaktischen Debatten an Universitäten und Schulen, aber auch im allgemeinen gesellschaftlichen Diskurs über Bildung.

(Antor 2007: 111; cf. Hallet/Königs 2010)

In meiner Arbeit erörtere ich den Einsatz einiger ausgewählter Texte zeitgenössischer Literatur von Autoren mit der Erfahrung beider Kulturen: der griechischen und der deutschen, auch im Hinblick auf Identitätsfindung und den komplexen Prozess kultureller Zugehörigkeit.

So erlebt Stella Bettermann in ihrer Erzählung Ich trink Ouzo, was trinkst du so? Meine griechische Familie und ich (2010) sowie in der Fortsetzung ${ }^{1}$ die griechische Kultur ihrer Mutter erst als Erwachsene bewusst, als sie sich einem Tanzkurs in München anschließt:

Wir schreiben März 2010, Griechenland steht vor dem Bankrott, und die deutsche Presse ist nicht gerade freundlich mit den Griechen umgesprungen. [...] es wird gestikuliert, geschimpft, ja geschrien. Und ich verstehe alles. Also zehn Prozent mehr, als ich in Griechenland verstehen würde. Das kommt daher, dass diese Griechen genauso sprechen wie meine Mama: Mischimaschi.

(Bettermann 2011:27)

Das Kulturthema Sprache zeigt sich, wie in diesem kurzen Beispiel, bei vielen der untersuchten Autoren als zentral; auch in der Beschreibung differierender Sitten und Gebräuche kulminieren die unterschiedlichen Auffassungen von Kultur, und hier äußern sich auch wechselseitige Stereotype. Und für diese Stereotype kann der/die Leser/in ein Bewusstsein entwickeln.

Doch ebenso bedeutsam ist nun die Entdeckung und Bewusstmachung der eigenkulturellen Prägungen sowie daraus hervorgehender kultureller Unterschiede und Gemeinsamkeiten. Der stetig wachsenden kulturellen Heterogenität Rechnung tragend soll interkulturelles Lernen im Fremdsprachenunterricht dabei die bisher zugrunde gelegte Dichotomie zwischen eigen und fremd auflösen und sowohl die kulturellen Einflüsse aller Schüler in den Lernprozess einbeziehen als auch die sich daraus ergebenden gegenwärtigen Bedürfnisse der Sprachlerner berücksichtigen.

(Eberhardt 2013: 21)

Zwar sieht der Gemeinsame Europäische Referenzrahmen vor, dass Sprachlerner sich mit solchen Themen auseinandersetzen und ein interkulturelles Bewusstsein entwickeln:

${ }^{1}$ Ich mach Party mit Sirtaki: Wie ich in Deutschland meine griechischen Wurzeln fand (2011). 
Als zweite Komponente interkultureller Kompetenz im GeR - neben dem soziokulturellen Wissen - findet interkulturelles Bewusstsein“ Erwähnung. Interkulturelles Bewusstsein meint hier ein Bewusstsein für die Beziehungen zwischen Herkunftsland und Zielsprachengemeinschaft, die durch Unterschiede und Ähnlichkeiten gekennzeichnet sind.

(Eberhardt 2013: 41)

Allerdings muss man konzedieren, dass sowohl in der didaktischen Literatur als auch in Curricula und der pädagogischen Praxis des Fremdsprachenunterrichts in Folge der Kompetenzorientierung die Beschäftigung mit literarischen Texten an den Rand gedrängt worden ist. „Der Beitrag von Literatur zur fremdsprachlichen Kompetenzentwicklung und zur Entfaltung der individuellen Schülerpersönlichkeit bleibt unberücksichtigt.“ (Hu 2008: 12)

\section{Praxisbeispiel: dialogisch}

In der Abteilung für Deutsche Sprache und Philologie der Aristoteles Universität Thessaloniki (wo ich 2007-2011 als DAAD- Lektorin tätig war) nahmen Studierende mit Erfahrungen sowohl in der griechischen wie in der deutschen Kultur (teilweise Remigranten) sowie einige Erasmus-Studierende an zwei Seminaren zu Fremdwahrnehmung und interkultureller Literatur teil, was zum doppelten Perspektivenaustausch einlud.

Die Studierenden erhielten in meinen literaturdidaktischen Seminaren einen Einblick in zeitgenössische deutschsprachige Literatur von Autoren mit Migrationshintergrund und erörterten mit meiner Hilfestellung theoretische Grundlagen der interkulturellen Germanistik bzw. kulturwissenschaftliche Sekundärliteratur über Stereotype und Fremdwahrnehmung und konnten selbst einen Literaturausschnitt aus dem von mir vorgeschlagenen Korpus wählen, den sie in einer schriftlichen Hausarbeit näher untersuchen wollten. Die Arbeit sollte drei Aspekte beinhalten: eine kurze Inhaltswiedergabe, persönliche Stellungnahme dazu sowie die Auseinandersetzung mit der Frage, ob und inwiefern dieser Ausschnitt sich für den Fremdsprachenunterricht eignen könnte (didaktische Perspektive), wobei auch die Sekundärliteratur zur Begründung herangezogen werden sollte. Die Studierenden kommentierten im Unterrichtsgespräch und in ihren Hausarbeiten meist persönlich oder anhand von Beispielen aus ihrem Erfahrungsumfeld, sehr lebendig.

Hier einige Ausschnitte aus Hausarbeiten zur Autorin Stella Bettermann, die sich auf unterschiedliche kulturelle Praktiken in Griechenland und Deutschland, im ersten Beispiel Religiosität, bezogen:

(1) „Meines Erachtens ist der Text geeignet für den Fremdsprachenunterricht. In dem Text werden die ,Unterschiede und Gemeinsamkeiten der griechischen und deutschen Kultur dargestellt. Aber es ist bedenklich, dass die Schüler vielleicht glauben, dass die Deutschen Atheisten seien, obwohl das gar nicht so ist. Es gibt nur sehr deutliche Unterschiede, was die Religion betrifft [...] Es ist möglich, dass Stereotype auftauchen, aber die Rolle des Lehrers ist es, die Unterschiede zwischen den jeweiligen Kulturen deutlich dazustellen. Dieser Text ist geeignet für Schüler, die sich in einem mittleren Niveau befinden (B1 bis B2).“ Die Studierenden haben ihn für die Didaktik der Landeskunde vorgeschlagen: „,...] Es werden nämlich zwei Kulturen nebeneinandergestellt.“ (M. M. Es werden Namenskürzel der Studierenden verwendet.) 
Hier wird deutlich, dass herkömmlicherweise kontrastiv gearbeitet wird, d. h. zwei Kulturen vergleichend nebeneinandergestellt werden.

Viele Gemeinsamkeiten und Identifikationsanlässe wurden von denjenigen Studierenden thematisiert, die griechische und deutsche Wurzeln hatten: Aufwachsen in zwei Kulturen, Sprachen und Religionen; Traditionen und Feste; Differenzen, Mentalitätsunterschiede; Kollision von Lebensentwürfen. Verschiedene kulturelle Praktiken und Nationalidentitäten wurden jedoch ebenfalls von Erasmus-Studierenden thematisiert, die gerade dabei waren, die griechische Kultur kennenzulernen. So schrieb beispielsweise eine Teilnehmerin griechischer Herkunft, die in Deutschland aufgewachsen ist:

(2) „Ich habe diesen Text ausgewählt, weil ich sehr viele Gemeinsamkeiten mit meinem eigenen Leben gefunden habe, ich bin auch Halbgriechin seitens meiner Mutter, und mein Vater war auch katholisch und ist aus steuerlichen Gründen aus der Kirche ausgetreten. Meine Geschwister und ich sind aber trotzdem griechisch orthodox getauft worden. Als ich in der Grundschule war, habe ich auch am Religionsunterricht teilgenommen, obwohl ich nicht katholisch war und bin jede Woche in die katholische Kirche gegangen, weil es für mich interessant war. Die Popen haben alles einfach dargestellt, im Gegensatz zur orthodoxen Kirche, bei der ich sowieso nicht so viel verstanden habe.“ (M. M.)

In den Hausarbeiten wurde auf traditionelle Feste und religiöse Praktiken sowie Aberglaube eingegangen. Eine andere Seminarteilnehmerin kommentierte die Häufung von typischen Gewohnheiten und Verhaltensweisen in der Erzählung wie folgt:

(3) „Man hat manchmal den Eindruck, dass es zu viel wird, weil keine Kleinigkeit weggelassen wird. Nicht alle Griechen sind so, wie es hier zum Vorschein kommt, nicht alle griechischen Mütter (wenn auch sehr viele!) möchten, dass sich der Tisch vor lauter Essen biegt, und nicht alle Mädchen in Deutschland sind fromm, immer sauber und frisch angezogen.“(E. R.)

Eine Erasmus-Studentin aus Deutschland, die ein Auslandssemester in Thessaloniki absolvierte, wies in Anlehnung an Bolten (2007: 59) auf die bereits im Seminar thematisierte Gefahr der Verfestigung von Stereotypen hin:

(4) „Es gilt als wichtige Aufgabe und Ziel der Lehrperson, Stereotype bewusst zu machen, sie zu differenzieren und in ihrer gefährlichen Funktion zu stigmatisieren. Der Text könnte hierbei eine veranschaulichende Rolle spielen, da zahlreiche Stereotype sowohl von Deutschen als auch von Griechen verwendet werden. Den Lernern muss gezeigt werden, dass Stereotype nicht unbedingt etwas Negatives bedeuten und nicht per se eine Misslingensbedingung für interkulturelle Kommunikation darstellen. Sie bieten eine wichtige Orientierung in der Wirklichkeit und vor allem in einer fremden Umgebung und dienen der Komplexitätsreduzierung.“ (K. W., Erasmus)

Die grundsätzliche Ambivalenz von Literatur - das haben die Studierenden erkannt - bietet sich in besonderer Weise zur Auseinandersetzung mit kulturell bedingten Perspektiven, Einstellungen und eben auch Stereotypen an. So äußerte eine Studentin:

(5) „Gerade weil literarische Texte zu unterschiedlichen Deutungen einladen und es ermöglichen, sich in Charaktere hineinzuversetzen und fremde Perspektiven zu erfahren, sind sie 
besonders dazu geeignet, eigene Vorstellungen über andere Kulturen zu überdenken und sogar zu relativieren. Desweiteren bedeutet eine Beschäftigung mit Literatur im Fremdsprachenunterricht eine Erweiterung des eigenen Horizontes. [...] Empathie und Ambiguitätstoleranz, die als Grundlage einer gelungenen interkulturellen Kommunikation gelten (Jürgen Bolten 2007: 74-75.) können mithilfe sorgfältig ausgewählter literarischer Texte gefördert werden und stellen somit eine große Hilfe bei der Entwicklung interkultureller Kompetenz dar.“ (K. W., Erasmus)

Aus eigener Erfahrung und aufgrund der Auseinandersetzung mit der entsprechenden Theorie zur Didaktik und Stereotypenforschung kam eine Studentin zu dem Schluss:

(6) „Wenn man eine Fremdsprache studiert, ist es unabdingbar, sich mit den Stereotypen und Vorurteilen des Landes auseinanderzusetzen, Literatur auszuwählen, die über dieses Problem berichtet, aber selbst keine Wertungen besitzen sollte, und zu diskutieren. Aus Erfahrung kann ich sagen, dass mir die Texte, die eine scharfen sarkastischen Humor haben, am besten gefallen, weil sie mit den Stereotypen spielen und uns aufgrund ihrer Kritik auffordern, darüber nachzudenken.“ (M. H., Erasmus)

Als positiv wurde auch die Tatsache gewertet, dass es sich um unterhaltsame und leicht zugängliche Literaturbeispiele handelte:

(7) „Bettermann legt ihren Fokus jedoch nicht auf die Unterhaltung, sondern vielmehr auf eine sympathische Vermittlung zwischen zwei Ländern, die in Zeiten der Krise auch miteinander in die Krise gerieten. [...] für mich als gebürtige Deutsche war es interessant zu sehen, wie die griechische Verwandtschaft im Buch auf mein Heimatland reagiert. Gleichzeitig fand ich mich selbst zwischen den Zeilen wieder und musste oft schmunzeln, wenn mir im griechischen Alltag Dinge widerfahren sind, welche Bettermann zuvor für mich im Buch erklärt hat.“(S. K., Erasmus)

Durch interkulturellen Unterricht kann Ethnozentrismus überwunden und die Bewusstmachung über Stereotype angeregt werden, wobei der dialogische Unterrichtsverlauf eine wichtige Rolle spielt (cf. Singer 2013: 43). Ziel meiner Moderation im Unterricht war also keineswegs die Vermeidung von Stereotypen und auch nicht deren Verfestigung, sondern die Bewusstmachung darüber, wie sie unsere Wahrnehmungen und unser sprachliches Handeln bestimmen und wie sie im Gespräch hinterfragt und möglicherweise aufgelöst werden können. Dies sollte m.E. Bestandteil eines zeitgemäßen Fremdsprachenunterrichts (zumal in heterogenen Lernkontexten) sein, der nicht bloß kulturkontrastiv, sondern auch mehrperspektivisch angelegt sein sollte und in dem Literatur ebenfalls eine Rolle spielt. Literatur und die Verständigung darüber eignen sich zur relativ freien Anwendung von sprachlichem Wissen; produktionsorientierte Ansätze bieten darüber hinaus idealerweise Gelegenheit zum selbstbestimmten Erproben sprachlichen Materials, was einen wesentlichen Beitrag zum Fremdsprachenlernen leistet.

\section{$6 \quad$ Ausblick}

Die hier aufgeführten studentischen Kommentare geben einen Eindruck davon, wie die Auseinandersetzung mit den gewählten Literaturausschnitten zunächst teilweise stark identifizie- 
rend, unhinterfragt (2) geschieht, aber auch - angeregt durch das Seminargespräch - die Auffälligkeit übertriebener Stereotypisierungen in den Texten kritisch kommentiert wird (3) und unterschiedlich stark differenzierte didaktische Konsequenzen abgeleitet werden $(1,4,6)$. Die Ambiguität und Deutungsvielfalt von Literatur wird dabei ebenfalls thematisiert (5) und die Rolle der Fremdsprachendidaktik im Hinblick auf Stereotype erörtert (6). Dabei wird auch deutlich, dass Humor eine positive Rolle im Umgang mit Stereotypen und bei der Entwicklung kultureller Kompetenz spielt $(6,7)$ und sich womöglich alltagsbezogene Erkenntnisse aus der Beschäftigung mit Literatur in interkultureller Perspektive ableiten lassen $(5,6,7)$.

Literaturdidaktische Zugänge, die weder auf einförmige Interpretationen abzielen, noch absolute Beliebigkeit im Umgang mit Literatur propagieren, sondern sich auf soziokulturelle Realien beziehen und die Lebenswelt der Teilnehmenden in einen dialogischen Prozess mit einbeziehen, scheinen am ehesten geeignet, eine Bewusstmachung über Stereotype anhand zeitgenössischer interkultureller Texte zu leisten.

In den deutschen Medien ist eine teilweise Verstärkung der Berufung auf Stereotype zu beobachten; Vokabeln wie ,Krisengriechen', ,faule Südländer', ,Lügenpresse“ (PEGIDA); ,Islamisierung' (dort und anderswo) sollen vordergründig auf den Punkt bringen, was aus einer diffusen Gemengelage von Halbwissen, Ressentiments, extremer Einzelfallbeobachtung, gröbster Verallgemeinerung und absichtlicher Diffamierung als gesellschaftliche ,Stimmung ‘ oder auch am sogenannten ,Stammtisch' geäußert wird. Durch die politische und vor allem mediale Instrumentalisierung solcher Stereotype gewinnen die Bürger allerdings nicht mehr Einsicht und Verständnis, sondern verstärken sich gegenseitige negative Zuschreibungen und Missverständnisse und werden die Grenzen zwischen ,fremd' und ,eigen' als unüberwindbar, verhärtet und konfliktträchtig interpretiert.

Im GeR werden die interkulturellen Kompetenzen den allgemeinen Kompetenzen zugeordnet. Wie man diese vermittelt, wird nicht eindeutig angegeben. Tagungen wie die aktuelle sind notwendig, um die Verständigung über Stereotype in allen Bereichen der Kommunikation auf eine bewusstere Ebene zu bringen, da man sie ohnehin nie völlig vermeiden kann (cf. Hallsteinsdóttir 2015).

Ein auf Dialog angelegter Unterricht, der Literatur zum Anlass nimmt, um ein Bewusstsein für kulturelle Unterschiede zu schaffen, ohne sich bloß auf Stereotype zu berufen, sondern diese kritisch zu betrachten, kann Anregungen zum Perspektivwechsel und Weckung von Empathie bieten sowie Reflexion über den Unterrichtszusammenhang hinaus in Gang setzen.

Dies ist eine der vorrangigsten Aufgaben für den interkulturellen Dialog.

\section{Literatur}

Addicks, Elisabeth et al. (2012): Stereotype Berichterstattung über ethnische Gruppen in deutschen Tageszeitungen. Erfurt: Friedrich-Ebert-Stiftung. http://library.fes.de/pdffiles/bueros/erfurt/09509.pdf [01.09.2015].

Albrecht, Corinna (2003): „Fremdheit“. In: Wierlacher, Alois/Bogner, Andrea (eds.): Handbuch interkulturelle Germanistik. Stuttgart, Metzler: 232-238. 
Auernheimer, Georg (s. a.): „Interkulturelle Kommunikation und Kompetenz“. http://jafriedrich.de/pdf/Interkulturelle\%20Kommunikation\%20und\%20Kompetenz.pdf [01.09.2015].

Bickes, Hans et al. (2012): Die Dynamik der Konstruktion von Differenz und Feindseligkeit am Beispiel der Finanzkrise Griechenlands: Hört beim Geld die Freundschaft auf? Kritisch-diskursanalytische Untersuchungen der Berichterstattung ausgewählter deutscher und griechischer Medien. Iudicium: München.

Bolten, Jürgen (2007): Interkulturelle Kompetenz. Erfurt: Landeszentrale für politische Bildung Thüringen.

Bredella, Lothar/Delanoy, Werner/Surkamp, Carola (eds.) (2004): Literaturdidaktik im Dialog. Tübingen: Narr.

Bredella, Lothar (2004): „Literaturdidaktik im Dialog mit Literaturunterricht und Literaturwissenschaft". In: Bredella, Lothar/Delanoy, Werner/Surkamp, Carola (eds.): Literaturdidaktik im Dialog. Tübingen, Narr: 21-64.

Bredella, Lothar (2008): „Ist interkulturelles Verstehen mit literarischen Texten möglich?“ In: Bosenius, Petra/Rohde, Andreas/Wolff, Martin (eds.): Verstehen und Verständigung. Interkulturelles Lehren und Lernen. Festschrift für Jürgen Donnerstag. Trier, WVT Wissenschaftlicher Verlag: 67-86.

Bredella, Lothar (2010): Das Verstehen des Anderen. Kulturwissenschaftliche und Literaturdidaktische Studien. Tübingen: Narr.

Cerri, Chiara (2009): „Interkulturelle Literatur“. In: Meurer, Petra/Ölke, Martina/Wilmes, Sabine (eds.): Interkulturelles Lernen. Mit Beiträgen zum Deutsch und DaF-Unterricht, zu ,Migranten '-Bildern in den Medien und zu Texten von Özdamar, Trojanow und Zaimoglu. Bielefeld, Aisthesis: 9-17.

Eberhardt, Jan-Oliver (2013): Interkulturelle Kompetenzen im Fremdsprachenunterricht. Auf dem Weg zu einem Kompetenzmodell für die Bildungsstandards. Trier: WVT wissenschaftlicher Verlag.

Esselborn, Karl (2010): Interkulturelle Literaturvermittlung. Zwischen didaktischer Theorie und Praxis. München: Iudicium.

Florack, Ruth (2007): Bekannte Fremde. Zur Herkunft und Funktion nationaler Stereotype in der Literatur. Tübingen: Niemeyer.

Gogolin, Ingrid (2007): „Interkulturelle Erziehung und das Lehren und Lernen fremder Sprachen“. In: Bausch, Karl-Richard/Christ, Herbert/Krumm, Hans-Jürgen (eds.): Handbuch Fremdsprachenunterricht. Tübingen, Narr: 96-102.

Hallet, Wolfgang/Königs, Frank G. (eds.) (2010): Handbuch Fremdsprachendidaktik. Seelze: Klett/Kallmeyer.

Hallsteinsdóttir, Erla (2012): Stereotype und interkulturelle Kompetenzen im dänischdeutschen Kontext (Anlage zum Projektantrag). www.stereotypenprojekt.eu/über-dasprojekt/hintergrund-des-projekts/ [01.09.2015].

$\mathrm{Hu}$, Adelheid et al. (2008): „Kompetenzorientierung, Bildungsstandards und fremdsprachliches Lernen - Herausforderungen an die Fremdsprachenforschung“. Zeitschrift für Fremdsprachenforschung 19/2: 163-186.

Ipsen, Guido (2009): „Erfahrungsbasierte Dekonstruktion von Stereotypen: Kulturelle Selbsterkenntnis als Spiegel des Anderen“. In: Meurer, Petra/Ölke, Martina/Wilmes, Sabine 
(eds.): Interkulturelles Lernen. Mit Beiträgen zum Deutsch- und DaF-Unterricht, zu ,Migranten'-Bildern in den Medien und zu Texten von Özdamar, Trojanow und Zaimoglu. Bielefeld, Aisthesis: 169-181.

Jonas, Kai J. (2002): Stereotypenentstehung im Intergruppenkontext. Dissertation an der Georg-August-Universität Göttingen. https://ediss.unigoettingen.de/bitstream/handle/11858/00-1735-0000-0006-B603-C/jonas.pdf?sequence=1 [01.09.2015].

Lammers, Karl Christian (2014): „Geschätzt, aber nicht geliebt. Die Wahrnehmung der deutschen Einheit in Dänemark". Deutschland Archiv 21.3.2014. www.bpb.de/181113 [01.09.2015].

Orosz, Magdolana (2010): „Interkulturalität, Identität, Fremdheit als Analysekategorien der Literatur der Frühen Moderne“. In: Hess-Lüttich, Ernest W. B. (ed.): Deutsch im interkulturellen Begegnungsraum Ostmitteleuropa. Frankfurt a. M. etc., Lang: 81-99.

Pieklarz, Madalena (2008): „Zur Erforschung von Stereotypen in der Fremdsprachendidaktik - ein geschichtlicher Überblick über Forschungsansätze und Darstellung eines Forschungsprojektes“. In: Chlosta, Christoph /Leder, Gabriela /Krischer, Barbara (eds.): Auf neuen Wegen. Deutsch als Fremdsprache in Forschung und Praxis. 35. Jahrestagung des Fachverbandes Deutsch als Fremdsprache 2007 an der Freien Universität Berlin. Göttingen, Universitätsverlag: 35-52.

Pümpel-Mader, Maria (2010): Personenstereotype. Eine linguistische Untersuchung zu Form und Funktion von Stereotypen. Winter: Heidelberg.

Reimer, Annett (2005): Die Bedeutung der Kulturtheorie von Geert Hofstede für das internationale Management. Wismar: Hochschule Wismar, Fachbereich Wirtschaft. www.wi.hswismar.de/ wdp/2005/0520_Reimer.pdf [01.09.2015].

Scheiffele, Eberhard (2000): „Affinität und Abhebung. Zum Problem der Voraussetzungen interkulturellen Verstehens“. In: Wierlacher, Alois (ed.): Das Fremde und das Eigene. Prolegomena zu einer interkulturellen Germanistik. München, Iudicium: 29-46.

Schmeling, Manfred (2004): „Multilingualität und Interkulturalität im Gegenwartsroman“. In: Schmitz-Emans, Monika (ed.): Literatur und Vielsprachigkeit. Heidelberg, Synchron: 222235.

Schugk, Michael (2004): Kulturbedingte Unterschiede in Verkauf und Werbung. München: Vahlen.

Singer, Gesa (2010): „Literarische und dialogische Annäherung an das ,Fremde‘. ,Fremdheit‘ in der deutschsprachigen Literatur als didaktisches Projekt“. IDV-Magazin 82: 489-501. www.idvnetz.org/publikationen/magazin/IDV-Magazin82.pdf [01.09.2015].

Singer, Gesa (2013): „Interkulturelle zeitgenössische Literatur: Eine Herausforderung für die Literaturwissenschaft". Estudios Filológicos Alemanes 26: 33-44.

Stratthaus, Bernd (2005): Was heißt interkulturelle Literatur? Dissertation an der Universität Duisburg-Essen. http://duepublico.uni-duisburgessen.de/servlets/DerivateServlet/Derivate15379/DISS_Was_heisst_interkulturelle_Literatur.pdf [01.09.2015].

Volkmann, Laurenz (2008): „Spracharbeit und language awareness im fremdsprachlichen Unterricht: Überlegungen zu einem vernachlässigten didaktischen Thema“. In: Bosenius, Petra/Rohde, Andreas/Wolff, Martin (eds.): Verstehen und Verständigung. Interkulturelles 
Lehren und Lernen. Festschrift für Jürgen Donnerstag. Trier, WVT wissenschaftlicher Verlag: 117-136. 